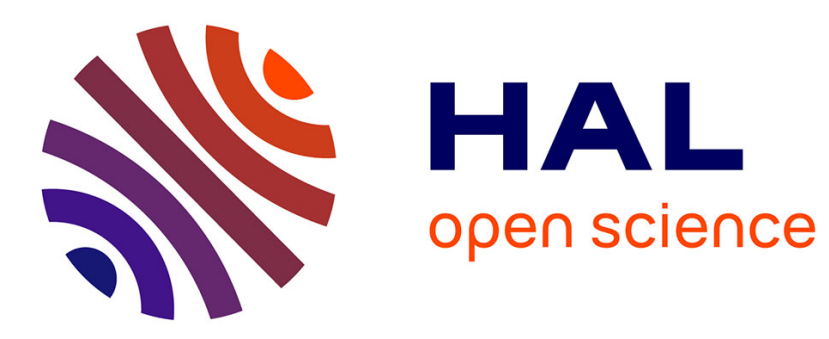

\title{
Medical ultrasound image reconstruction using distributed compressive sampling
}

Adrian Basarab, Hervé Liebgott, Olivier Bernard, Denis Friboulet, Denis

Kouamé

\section{- To cite this version:}

Adrian Basarab, Hervé Liebgott, Olivier Bernard, Denis Friboulet, Denis Kouamé. Medical ultrasound image reconstruction using distributed compressive sampling. IEEE International Symposium on Biomedical Imaging: From Nano to Macro - ISBI 2013, Apr 2013, San Francisco, United States. pp. 628-631. hal-01150347

\section{HAL Id: hal-01150347 \\ https://hal.science/hal-01150347}

Submitted on 11 May 2015

HAL is a multi-disciplinary open access archive for the deposit and dissemination of scientific research documents, whether they are published or not. The documents may come from teaching and research institutions in France or abroad, or from public or private research centers.
L'archive ouverte pluridisciplinaire HAL, est destinée au dépôt et à la diffusion de documents scientifiques de niveau recherche, publiés ou non, émanant des établissements d'enseignement et de recherche français ou étrangers, des laboratoires publics ou privés. 


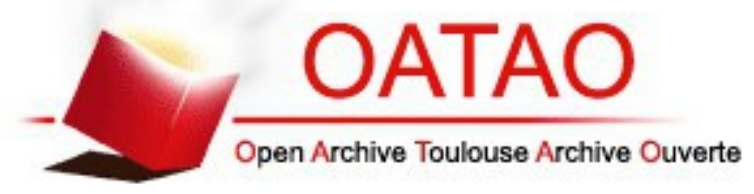

\section{Open Archive TOULOUSE Archive Ouverte (OATAO)}

OATAO is an open access repository that collects the work of Toulouse researchers and makes it freely available over the web where possible.

This is an author-deposited version published in : http://oatao.univ-toulouse.fr/ Eprints ID : 12355

To link to this article : DOI :10.1109/ISBI.2013.6556553

URL : http://dx.doi.org/10.1109/ISBI.2013.6556553

To cite this version : Basarab, Adrian and Liebgott, Hervé and Bernard, Olivier and Friboulet, Denis and Kouamé, Denis Medical ultrasound image reconstruction using distributed compressive sampling. (2013) In: IEEE International Symposium on Biomedical Imaging: From Nano to Macro - ISBI 2013, 7 April 2013 - 11 April 2013 (San Francisco, United States).

Any correspondance concerning this service should be sent to the repository administrator: staff-oatao@,listes-diff.inp-toulouse.fr 


\title{
MEDICAL ULTRASOUND IMAGE RECONSTRUCTION USING DISTRIBUTED COMPRESSIVE SAMPLING
}

\author{
Adrian Basarab ${ }^{1}$, Hervé Liebgott ${ }^{2}$, Olivier Bernard ${ }^{2}$, Denis Friboulet $^{2}$, Denis Kouamé ${ }^{1}$ \\ ${ }^{1}$ Université de Toulouse, IRIT, CNRS UMR 5505, Université Paul Sabatier, Toulouse, France \\ ${ }^{2}$ Université de Lyon, CREATIS, CNRS UMR 5220, INSERM U630, Université de Lyon 1, INSA-Lyon, \\ Lyon, France
}

\begin{abstract}
This paper investigates ultrasound (US) radiofrequency (RF) signal recovery using the distributed compressed sampling framework. The "correlation" between the RF signals forming a RF image is exploited by assuming that they have the same sparse support in the 1D Fourier transform, with different coefficient values. The method is evaluated using an experimental US image. The results obtained are shown to improve a previously proposed recovery method, where the correlation between RF signals was taken into account by assuming the 2D Fourier transform of the RF image sparse.
\end{abstract}

Index Terms - ultrasound imaging, compressive sampling, jointly sparse signal, radiofrequency signals, Fourier transform.

\section{INTRODUCTION}

Ultrasound (US) imaging is one of the most commonly used medical imaging modalities. Its low cost, non-ionizing characteristics, ease of use and real-time nature make it the gold standard for many crucial diagnostic exams, especially in obstetrics and cardiology. However, this real-time property is sometimes limited by the intrinsic acquisition time or volume of data, especially in 3D imaging. Even in 2D US imaging, specific applications, such as cardiac US monitoring or small animal experiments where speckle decorrelation in time is an issue, would also benefit from an increased frame rate.

In this context, a few research teams have recently started to investigate the application of the compressed sampling (CS) [1] framework to ultrasound imaging. CS is a recent and very promising theory allowing the reconstruction of signals and images from relatively few acquired samples (below Shannon-Nyquist's criteria). Based on two key conditions, i) the information has to be sparse in a known basis, ii) the measurements have to be incoherent with this basis, CS ensures an exact reconstruction with overwhelming high probability via $L_{l}$ convex constrained optimization.

In US imaging, the potential of CS was evaluated at different stages of the image formation. In [2], the reconstruction of raw radiofrequency (RF) signals (signals received by each element of the US probe) is proposed, based on the sparsity assumption in the waveatoms domain. In [3], the CS framework is integrated in the beamforming process, considering the US image itself being sparse. In [4], the CS theory is used to reconstruct bandlimited RF images and a random acquisition scheme allowing the reduction of US wave emissions is proposed. In [5,6], $L_{l}$-based US image reconstruction methods are employed in order to enhance the resolution of high frame rate images obtained within the plane wave technique. For a detailed review and further details concerning the application of CS framework in US imaging, the reader may refer to [7].

In this paper, we consider the problem of post-beamforming $\mathrm{RF}$ image reconstruction from a few randomly chosen samples. In [4], the same issue was addressed, and the reconstruction was done by minimizing the $L_{l}$ norm of the 2D Fourier transform of the RF image, considered sparse therein. This paper focalizes on RF signal reconstruction based on a different assumption, of jointly sparse [8] or group sparse [9] signals. Herein, we proposed to jointly reconstruct the RF signals forming the RF image, using the framework of distributed compressed sensing (DCS) proposed in [8]. In [8], the reconstruction of an ensemble of jointly sparse signals is addressed and theoretical and experimental results show the advantages of taking into account such an assumption. In our work, we use the model that considers that all the signals to reconstruct have the same sparse support (in our case in the 1D Fourier domain), but with different coefficients. This hypothesis seems reasonable for US imaging, considering that the RF signals are bandlimited by the impulse response of the scanner.

We should note that the same assumption of jointly sparse RF signals in the Fourier domain has already been exploited in US imaging in [6]. However, the purpose of the method proposed in [6] is different from several points of view. First, in [6] the measurements are not samples at random positions as in our case, but projections of raw RF signals on Gaussian random vectors. Second, the purpose of [6] is to reconstruct deconvolved images. Thus, the PSF of the system is considered to be known and spatially invariant. In our work, RF images are reconstructed without any knowledge about the PSF (except that it is bandlimited). 
Third, in [6], simulated raw signals obtained by plane wave imaging are exploited, corresponding to a medium with only a few scatterers. In our work, results on an experimental image are presented, obtained using classical US wave emission-reception and beamforming.

In the following sections, our method is described and results on an in vivo image are provided.

\section{PROBLEM FORMULATION}

Let us denote by $\boldsymbol{X} \in \mathbb{R}^{N \times J}$ an US RF image formed by $J$ RF signals of length $N$ samples, denoted by $\boldsymbol{x}_{\boldsymbol{1}}, \boldsymbol{x}_{2}, \ldots, \boldsymbol{x}_{\boldsymbol{J}}$. Moreover, we denote by $\boldsymbol{\theta}_{j} \in \mathbb{C}^{N}$ the 1D Fourier transforms of $\boldsymbol{x}_{\boldsymbol{j}}$, for $j$ running from 1 to $J$.

$$
\boldsymbol{\theta}_{\boldsymbol{j}}=\boldsymbol{F} \boldsymbol{x}_{\boldsymbol{j}}, \quad j \in\{1,2, \ldots, J\}
$$

The $J$ RF signals are considered jointly $S$-sparse in the 1D Fourier domain. That means that all $\boldsymbol{\theta}_{\boldsymbol{j}}$ have $S$ non zeros elements, at the same unknown positions, but with different values (in practice, RF signals may be prefiltered if needed). In our framework, we consider $M<<N$ measurements for each RF signal, by randomly decimating $\boldsymbol{x}_{\boldsymbol{j}}$. We denote by $\boldsymbol{y}_{\boldsymbol{j}} \in \mathbb{R}^{\boldsymbol{M}}$ the measurement vector corresponding to $\boldsymbol{x}_{\boldsymbol{j}}$.

$$
\boldsymbol{y}_{\boldsymbol{j}}=\boldsymbol{\varphi}_{\boldsymbol{j}} \boldsymbol{x}_{\boldsymbol{j}}, \quad j \in\{1,2, \ldots, J\}
$$

Where $\boldsymbol{\varphi}_{j} \in \mathbb{R}^{\boldsymbol{M} \times \boldsymbol{N}}$ are matrices obtained by taking randomly $M$ lines from the identity matrix of size $N \times N$.

By replacing (1) in (2), we obtain:

$$
\boldsymbol{y}_{\boldsymbol{j}}=\boldsymbol{\varphi}_{\boldsymbol{j}} \mathcal{F}^{-1} \boldsymbol{\theta}_{\boldsymbol{j}}=\boldsymbol{A}_{\boldsymbol{j}} \boldsymbol{\theta}_{\boldsymbol{j}}, \quad j \in\{1,2, \ldots, J\}
$$

With $\boldsymbol{A}_{j} \in \mathbb{C}^{\boldsymbol{M} \times \boldsymbol{N}}$ matrices requiring the conditions imposed by CS framework.

In our previous work $[2,4]$, based on the assumption that the 2D Fourier transform of $\boldsymbol{X}$ is sparse, we recovered the RF image using the following optimization:

$$
\underset{X}{\operatorname{Argmax}}\left(\|\Phi X-Y\|_{2}+\lambda\left\|\mathcal{F}_{2 D}(X)\right\|_{1}\right)
$$

Where $\boldsymbol{\Phi}$ is a random decimation mask (corresponding to $\left.\boldsymbol{\varphi}_{\boldsymbol{j}}\right), \boldsymbol{Y}$ are the measured sample (corresponding to vectors $\left.\boldsymbol{y}_{\boldsymbol{j}}\right), \boldsymbol{F}_{2 \boldsymbol{D}}$ is the $2 \mathrm{D}$ Fourier operator and $\boldsymbol{\lambda}$ is a parameter weighting between sparsity and data fidelity. Note that the parameter $\lambda$ was tuned using a re-weighted iterative technique [10], together with a classical conjugate gradient descent.

As explained in the introduction, we propose in this paper a different way of reconstructing the RF image $\boldsymbol{X}$, by jointly recovering the RF signals $\boldsymbol{x}_{\boldsymbol{j}}$. Thus, the assumption of sparse
2D Fourier transform is replaced herein by the assumption of RF signals being sparse and having the same support (with different values) in the 1D Fourier domain. In the results section we compare our proposal to those obtained by solving (4).

\section{JOINTLY SPARSE SIGNAL RECONSTRUCTION}

In this section, we give the main details of the recovery algorithm used to retrieve the $J$ 1D Fourier transforms $\boldsymbol{\theta}_{\boldsymbol{j}}$ from the $J$ measurement vectors $\boldsymbol{y}_{\boldsymbol{j}}$. In this scope, we use a modified version of the Orthogonal Matching Pursuit algorithm adapted to jointly sparse signals $[8,11]$. In the following, the main steps of the algorithm are given. The variable $k$ indicates the algorithm iterations, starting for $1(k$ is equal to 0 at the initialization step).

Note: The indices between parentheses indicate the iteration number.

Step 1 - initialization

Initialize all Fourier transforms corresponding to the $J$ signals to $\mathbf{0}$ vectors:

$$
\widehat{\boldsymbol{\theta}}_{\boldsymbol{j}}(\mathbf{0})=\mathbf{0}
$$

Initialize the residuals to $\boldsymbol{y}_{\boldsymbol{j}}-\boldsymbol{A}_{\boldsymbol{j}} \widehat{\boldsymbol{\theta}}_{\boldsymbol{j}}$ :

$$
\boldsymbol{r}_{\boldsymbol{j}}(\mathbf{0})=\boldsymbol{y}_{\boldsymbol{j}} \text {, with } \boldsymbol{r}_{\boldsymbol{j}} \in \mathbb{C}^{M}
$$

Initialize the matrices containing the selected atoms (after orthogonalization):

$$
\widetilde{A}_{j}(\mathbf{0})=\mathbf{0}
$$

Step 2 - selection of the atom that maximizes the sum of the magnitudes of the projection of the residuals on the sampling matrices

$$
n_{k}=\underset{n=1, \ldots, N}{\operatorname{Argmax}} \sum_{j=1}^{J} \frac{\left|\left\langle r_{j}(k-1), A_{j, n}\right\rangle\right|}{\left\|A_{j, n}\right\|^{2}}
$$

where the vector $\boldsymbol{A}_{\boldsymbol{j}, \boldsymbol{n}}$ stands for n-th column of matrix $\boldsymbol{A}_{j}$.

Add the index of the new detected atom to the set of selected indices: $\boldsymbol{\Omega}=\left[\boldsymbol{\Omega} \boldsymbol{n}_{\boldsymbol{k}}\right]$

Extract the new atom:

$$
a_{j}(k)=A_{j, n_{k}}
$$

Step 3 - orthogonalize the new selected atom against the previous orthogonalized selected atoms

$$
\widetilde{a}_{j}(k)=a_{j}(k)-\sum_{l=0}^{k-1}\left[\left\langle a_{j}(k), \widetilde{a}_{j}(k-1)\right\rangle \cdot a_{j}(k)\right]
$$

Normalize the new selected vector 


$$
\widetilde{\boldsymbol{a}}_{j}(\boldsymbol{k})=\frac{\widetilde{\boldsymbol{a}}_{j}(\boldsymbol{k})}{\left\|\widetilde{\boldsymbol{a}}_{j}(\boldsymbol{k})\right\|_{2}^{2}}
$$

Step 4 - Update $\widehat{\boldsymbol{\theta}}_{\boldsymbol{j}}$ taking into account the new selected vector

$$
\widehat{\boldsymbol{\theta}}_{j}(\boldsymbol{k})=\left\langle\widetilde{\boldsymbol{A}}_{j}(\boldsymbol{k}), \boldsymbol{r}_{j}(\boldsymbol{k}-\mathbf{1})\right\rangle
$$

Where $\widetilde{\boldsymbol{A}}_{j}(\boldsymbol{k})$ is the matrix which columns are the $k$ selected orthogonalized vectors, corresponding to signal $j$.

Step 5 - Update the residuals

$$
r_{j}(k)=r_{j}(k-1)-\widetilde{A}_{j}(k) \cdot \widehat{\theta}_{j}(k)
$$

Step 6 - Check for convergence

If one of the following conditions is achieved, then go to step 7

- $\quad r_{j}(\boldsymbol{k})$ is larger than a give threshold for all signals $j$

- $\quad \mathrm{k}$ is equal to $\mathrm{M}$

- the residual is increasing for at least one signal

Otherwise, go to step 2.

Step 7 -De-orthogonalize the solution

See [11] for more details about the need of this step.

Step 8 - Find the estimated RF signals

$$
\widehat{\boldsymbol{x}}_{\boldsymbol{j}}=\mathcal{F}^{-1} \widehat{\boldsymbol{\theta}}_{\boldsymbol{j}}, \quad j \in\{1,2, \ldots, J\}
$$

\section{RESULTS}

In this section we show comparative results between the proposed approach and the method recovering the RF image by minimizing (4). The reconstruction results are obtained from an in vivo image of a human healthy thyroid. The image was acquired using a clinical scanner (Sonoline Elegra) using a $7.5-\mathrm{MHz}$ linear probe (Siemens Medical Systems, Issaquah, WA, USA). The RF lines were bandpass filtered and sampled at $50 \mathrm{MHz}$.

In our experiments, we cropped the image to a $256 \times 2048$ region, which resulted in $256 \mathrm{RF}$ signals of 2048 samples each $(J=256, N=2048)$. Taking into account the spectral content of each RF signal, we can consider their 1D Fourier transforms S-sparse, with S equal to 500 samples.

The $256 \mathrm{RF}$ signals were randomly decimated as shown in (2). In [8], it is suggested that for a large number of signals $(J \rightarrow \infty),(S+1)$ measures per signal suffice for a "perfect" reconstruction. In our case, as in all practical experiments, we dispose of a limited number of signals (256). We show in Figure 1 the evolution of the reconstruction normalized root mean square error (NRMSE) between the real image and the reconstructed one using the method described in section 3, for different number of measurements $M$. We observe that in this case, 600 samples per signal are sufficient for a "perfect" recovery.

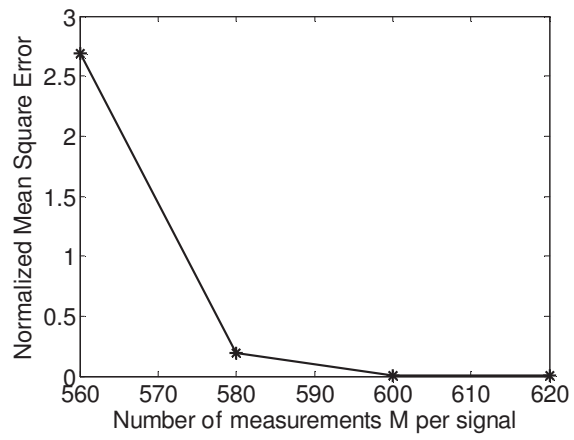

Figure 1. Reconstruction using the proposed method. Recovery normalized root mean square error (NRMSE) evolution for varying number of measurements per signal.

Note that if we replace the measurements matrices $\boldsymbol{A}_{j}$ in equation (3) by random Gaussian matrices, as suggested in [6], then taking 501 measurements per signal would be sufficient in our case (experimentally verified) for a "perfect" recovery. However, we consider that this way of measuring the RF signals is less interesting in practice.

Note also that in the particular case presented herein, a separate reconstruction of each RF signal using the same optimization algorithm requires 750 samples per signal for a "perfect" recovery.

In the following, we compare the results obtained with the proposed method to those obtained by minimizing (4). For this, we show the recovery results obtained for both methods using $256 \times 600$ random samples. As explained previously, for this decimation rate, the reconstruction with the proposed DCS method is "perfect", in the sense that the order of magnitude of the reconstruction error is $10^{-12}$. We show in Figure 2 the $\mathrm{B}$ mode images corresponding to the true and reconstructed RF images. We observe that the method minimizing the $L_{l}$ norm of the 2D Fourier transform provides a noisier result. The NRMSE corresponding to this result is 0.29 (the amplitude of the RF signals are normalized between -1 and 1 ).

In order to better highlight the differences between the two results, we show in Figure 3 a zoom on a small region extracted from the three images in Figure 2.

In Figure 4, we show the random samples corresponding to a local region extracted from one RF line and the recovery results obtained with both methods. As explained previously, with our method the NRMSE is roughly $10^{-12}$. For this reason, we only show the recovered RF lines, as the true RF line is identical to our result. 


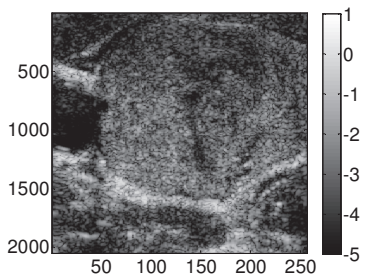

(a)

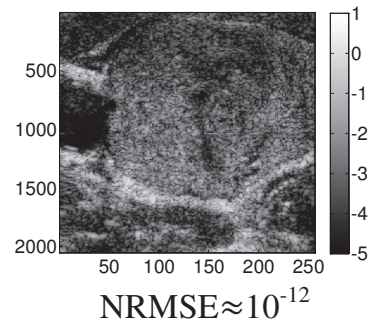

(b)

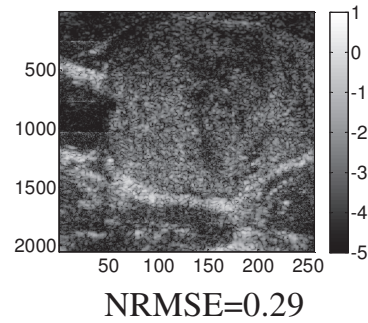

(c)
Figure 2. (a) B-mode image corresponding to the true RF image, (b) and (c) B-mode images corresponding to the recovered RF images with the proposed method and by minimizing (4).

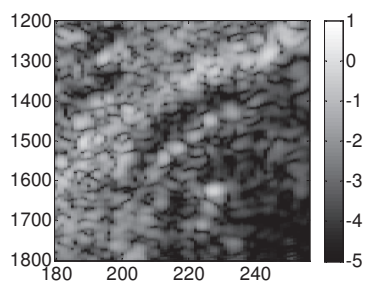

(a)

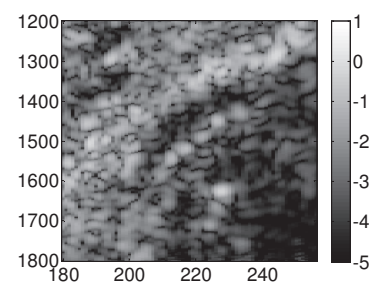

(b)

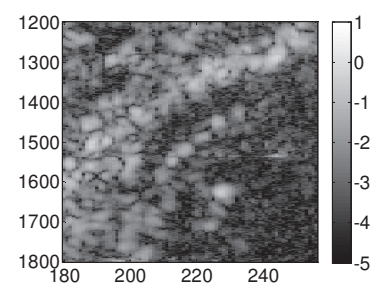

(c)
Figure 3. Local regions extracted from Figure 2(a,b,c).

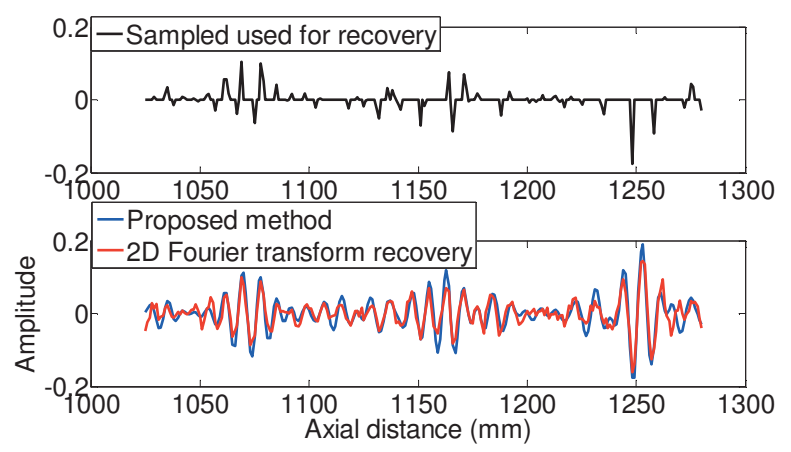

Figure 4. Top plot: Random samples used for CS reconstruction, for a local region of a RF line (the discarded samples are forced to $0)$. Bottom plot: recovered RF lines for both methods. The true initial RF line is identical to the one recovered with our method.

\section{CONCLUSION}

In this paper, we proposed to use the framework of distributed compressive sampling for RF line recovery in ultrasound imaging. For this, we assumed that the RF lines forming a RF image have the same sparse support in the 1D Fourier domain, with different magnitudes and phases. We have shown that the results are more accurate, for the same number of measurements, than those obtained by minimizing the $L_{l}$ norm of the 2D Fourier transform of the $\mathrm{RF}$ image. In this work, the measurements are considered samples of the RF image taken at random positions. However, as discussed in our previous work [4], for ultrasound imaging it is more convenient to reduce the number of RF lines, by randomly skipping part of them. In future work, we will evaluate the application of DCS in this context, by jointly recovering several RF images.

\section{REFERENCES}

[1] E.J. Candes, J. Romberg and T. Tao, "Robust uncertainty principles: exact signal reconstruction from highly incomplete frequency information," IEEE Transactions on Information Theory, vol. 52, no. 2, pp. 489-509, 2006.

[2] H. Liebgott, R. Prost, and D. Friboulet, "Pre-beamformed RF signal reconstruction in medical ultrasound using compressive sensing," Ultrasonics, in press, 2012.

[3] N. Wagner, Y. C. Eldar and Z. Friedman, "Compressed Beamforming in Ultrasound Imaging", IEEE Transactions on Signal Processing, vol. 60, no. 9, pp.4643-4657, 2012.

[4] C. Quinsac, A. Basarab, and D. Kouamé, "Frequency domain compressive sampling for ultrasound imaging," Advances in Acoustics and Vibration, special issue on Advances in Acoustic Sensing, Imaging, and Signal Processing, vol. 12, pp. 1-16, 2012.

[5] M. Schiffner, T. Jansen, and G. Schmitz, "Compressed Sensing for Fast Image Acquisition in Pulse-Echo Ultrasound," Biomedical Engineering, vol. 57, pp. 192-195, 2012.

[6] Q. Zhang, B. Li, and M. Shen, "A measurement domain adaptive beamforming approach for ultrasound instrument based on distributed compressed sensing: Initial development," Ultrasonics, in press, 2012.

[7] H. Liebgott, A. Basarab, D. Kouamé, O. Bernard, D. Friboulet, "Compressive sensing in medical ultrasound," IEEE Ultrasonics Symposium, in press, 2012.

[8] M.F. Duarte, S. Sarvotham, D. Baron, M.B. Wakin, R.G. Baraniuk, "Distributed compressed sensing of jointly sparse signals," Asilomar Conf. Signals, Sys., Comput, pp. 1537-1541, 2005.

[9] W. Deng, W. Yin, and Y. Zhang, "Group sparse optimization by alternating direction method," TR11-06, Department of Computational and Applied Mathematics, Rice University, 2011.

[10] E. J. Candes, M. B.Wakin, and S. P. Boyd, "Enhancing sparsity by reweighted _1 minimization," Journal of Fourier Analysis and Applications, vol. 14, no. 5-6, pp. 877-905, 2008.

[11] J. Tropp, A.C. Gilbert, M.J. Strauss, "Simultaneous sparse approximation via greedy pursuit," IEEE ICASSP, March 2005. 